\title{
Bacteriological spectrum and immediate outcome of neonatal sepsis in tertiary care centre in South India
}

\author{
Sahoo M.R ${ }^{1}$, Arigela $V^{2}$, A Sirisha ${ }^{3}$ \\ ${ }^{1}$ Dr Manas Ranjan Sahoo, Associate Professor, ${ }^{2}$ Dr Vasundhara Arigela, Professor, ${ }^{3}$ Dr A Sirisha, Junior \\ Resident; authors are attached with Department of Pediatrics, Alluri Sitaramaraju Academy of Medical \\ Sciences, Malkapuram, West Godavari, NH-5, Vijayawada Visakapatnam Road, Eluru, Eluru, Andhra Pradesh \\ 534005, India.
}

Address for Correspondence: Dr Manas Ranjan Sahoo, Email: drmrsahoo@gmail.com

\begin{abstract}
Introduction: Neonatal septicemia is diagnosed when generalized systemic features of sepsis are associated with pure growth of bacteria from one or more sites. It refers to systemic infection of neonates including Septicemia, Pneumonia, Meningitis, Arthritis, Osteomyelitis and UTI. Objective: To study the bacteriological profile and antibiotic sensitivity of neonatal sepsis and correlating to outcome. Materials and Methods: This is a prospective hospital based study in NICU of ASRAM medical college, Eluru. Period of study: January, 2014 to July, 2015 Selection of cases: 74 Neonates below the age of 28 days with clinical suspicion of neonatal septicemia were included in this study. Results: Early onset septicemia was present in 49 cases $(66 \%)$, Late onset septicemia was present in 25 cases (34\%). E-coli, Klebsiella and CONS were the most common organisms isolated. Present study shows Gram negative organisms show maximum sensitivity to Imipenem (87.5\%). Gram negative organisms show least sensitivity to cefotaxime (25\%). Conclusion: Early-onset septicemia is more common than late-onset septicemia. Gram-negative septicemia is more common than Gram-positive septicemia. Gram-negative organisms are common cause of early-onset septicemia. Mortality is higher in early-onset septicemia and Gram-negative septicemia.
\end{abstract}

Keywords: Bacteriological profile; early onset sepsis; late onset sepsis; neonate

\section{Introduction}

Neonatal septicemia is diagnosed when generalized systemic features of sepsis are associated with pure growth of bacteria from one or more sites [1]. It refers to systemic infection of neonates including Septicemia, Pneumonia, Meningitis, Arthritis, Osteomyelitis and UTI. Systemic bacterial infection during the first month of life has remained a major cause of neonatal morbidity and mortality despite the development of broad spectrum antimicrobial agents and technological advancements in life supportive therapy. The early diagnosis of neonatal septicemia still poses great difficulties. Early clinical symptomatology of neonatal septicemia is mimicked by lot of other disorders affecting the newborn. Neonatal sepsis can be divided into two subtypes depending upon

Manuscript received: $5^{\text {th }}$ June 2017

Reviewed: $15^{\text {th }}$ June 2017

Author Corrected: $24^{\text {th }}$ June 2017

Accepted for Publication: $30^{\text {th }}$ June 2017 whether the onset of symptoms is during the first 72 hours of life or later. Although the term early onset sepsis had been used to refer to neonatal infections occurring as late as one week of age, it should be restricted to those infections with a perinatal pathogenesis, the usual onset of which occur within 72 hours. Early-onset sepsis is caused by organisms prevalent in genital tract or in the labor room. Ascending infection, trans placental and hematogenous spreads are important mechanisms of early-onset sepsis.

After the birth the baby is exposed to the environment contaminated with microorganisms, which start settling or colonizing at various organ system. The organisms enter the body through the umbilicus, skin or mucosa. Due to poor immunological defense of the new born, even local infections tend to become generalized. Infections 
are more commonly seen with preterm and low birth weight babies. To prevent serious morbidity and mortality early diagnosis and timely intervention are mandatory.

The incidence of neonatal sepsis from the National Neonatal Perinatal Database (NNPD 2002-03) is 30 per 1000 live births (3\% intramural and 39.7\% among extramural babies).

The database comprising 18 tertiary-care neonatal units across India found sepsis to be one of the commonest causes of neonatal mortality, contributing to $19 \%$ of all neonatal deaths. In India, sepsis contributes to up to $52 \%$ of neonatal deaths in the community and $36 \%$ of deaths in hospitalized newborns. Literature shows that septicemia was the commonest clinical category with an incidence of 23 per 1000 live births [2].

Meningitis occurs in $2-5 \%$ of neonates with suspected sepsis and approximately $25 \%$ of culture proven sepsis. The incidence and mortality is much higher in very low birth weight (VLBW) neonates compared to term neonates.

\section{Aims and Objectives}

To study the bacteriological profile and antibiotic sensitivity of neonatal sepsis and correlating to outcome

\section{Materials and Methods}

This is a prospective hospital based study in NICU of ASRAM medical college, Eluru.

Period of study: January, 2014 to July, 2015

Selection of cases: 74 Neonates below the age of 28 days with clinical suspicion of neonatal septicemia were included in this study. Neonates admitted in our hospital from outpatient department and neonates born in our hospital were included in this study group.

After admission detailed history was taken and thorough clinical examination was done. These neonates had the following symptoms and signs which were suspicious of septicemia.

Symptoms: Fever, Refusal of feeding, Lethargy, Respiratory Distress, Vomiting, Abdominal distension, Irritability, Rash.
Signs: Hypothermia, Hyperthermia, Apnea, Pallor, Jaundice, Sclerema, petechiae, purpura, shock.

All neonates were investigated as follows:-

\section{Sepsis Screen}

1. Total leucocyte count was done by using Neubers chamber. Leukopenia with count $<$ 5000cells / cumm was considered positive for septicemia.

2. Peripheral smear prepared with a drop of blood from heel prick and stained with Leishman's stain. The neutrophil is about 10-12 microns in diameter. The cytoplasm contains fine pale violet granules and number of nuclear lobes increase with maturity. Toxic granules were identified as a coarse darkly stained granules and its percentage was calculated.

3. Micro-ESR: Is simple and inexpensive. It was obtained by collecting capillary blood in standard pre-heparinized micro hematocrit tube of $75 \mathrm{~mm}$ length, $1.1 \mathrm{~mm}$ internal diameter and $1.5 \mathrm{~mm}$ outer diameter. Fall of erythrocyte column was read after one hour. During neonatal period a value of more than $15 \mathrm{~mm}$ at the end of $1 \mathrm{st} \mathrm{hr}$. was considered as suggestive of infection.

4. C-reactive protein: C-reactive protein was detected by latex agglutination test. CRP $\geq 2.4 \mathrm{mg} / \mathrm{dl}$ was taken as positive.

5. Blood Culture: In all neonates the blood sample was collected from peripheral vein with all aseptic precautions, prior to administration of any antibiotic therapy. $0.5 \mathrm{ml}$ of blood was collected in $5 \mathrm{ml}$ of glucose broth.

This sample was immediately sent to Microbiology Department, three subcultures were observed after 24, 48 and 120 hrs. If no growth was observed after five days, culture was reported as negative. If growth was observed material was further analyzed for specific organisms.

Gram negative organisms showed characteristic colonies on nutrient agar and MacConkey medium. E Coli on MacConkey medium showed pink colonies.

On nutrient agar Staphylococci showed colonies of different colours. 
Original Research Article

\section{Results}

This study was conducted in Alluri SitaRama Raju Academy of Medical Sciences, Malkapuram, Eluru during study period of one and half year. 74 neonates below the age of 28 days with clinical suspicion of neonatal septicemia were included in this study.

Early onset septicemia was present in 49 cases (66\%), Late onset septicemia was present in 25 cases (34\%). Early onset septicemia was more common than late onset septicemia. Culture was bacteriologically negative in 62 cases.

Table No-1: Organisms isolated in culture positive cases.

\begin{tabular}{|c|c|}
\hline Name of the organism isolated & No. of cases \\
\hline E Coli & 4 \\
\hline Klebsiella & 3 \\
\hline CONS & 1 \\
\hline COPS & 1 \\
\hline Moraxella & 3 \\
\hline
\end{tabular}

E-coli, klebsiella and CONS were the most common organisms isolated. Other organisms isolated were COPS and Moraxella. Gram-negative organisms were detected in 8 cases. Gram-positive organisms were detected in 4 cases. Gram-negative septicemia was more common than Gram-positive septicemia.

Table No.-2: Distribution of organisms isolated according to age of onset of Septicemia.

\begin{tabular}{|c|c|c|c|}
\hline & \multicolumn{2}{|c|}{ Gram staining } & Total \\
\hline$<72 \mathrm{hrs}$ & Gram-negative & Gram-positive & 8 \\
\hline$($ EOS $)$ & & & \\
\hline$>72 \mathrm{hrs}$ & 6 & 2 & 4 \\
\hline$($ LOS $)$ & 2 & $\mathbf{4}$ & $\mathbf{1 2}$ \\
\hline Total & $\mathbf{8}$ & & \\
\hline
\end{tabular}

Out of 8 culture positive cases of early onset septicemia, Gram-negative organisms were detected in 6 cases , Gram-negative organisms were common cause of early onset septicemia.

Table No 3 (A):- Antimicrobial sensitivity pattern of Gram Negative isolates.

\begin{tabular}{|c|c|c|c|c|c|c|c|}
\hline$\underset{\dot{0}}{\dot{0}}$ & 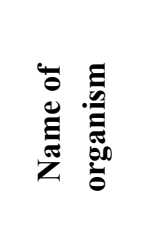 & 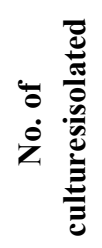 & 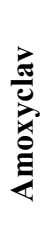 & & 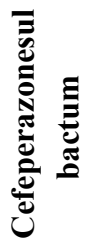 & & 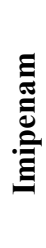 \\
\hline 1 & E.coli & 4 & 1 & 2 & 3 & 1 & 3 \\
\hline 2 & Klebsiella & 3 & 1 & 2 & 2 & - & 3 \\
\hline 3 & Moraxella & 1 & 1 & - & 1 & 1 & 1 \\
\hline & Total & 8 & 3 & 4 & 6 & 2 & 7 \\
\hline
\end{tabular}

Observation:-Present study shows Gram negative organisms show maximum sensitivity to Imipenam, followed by cefeperazone sulbactam, Amikacin, Amoxyclav. Gram negative organisms show least sensitivity to cefotaxime. 
Table No- 3 (B): Antimicrobial sensitivity pattern of Gram positive organisms

\begin{tabular}{|c|c|c|c|c|c|c|c|c|c|c|c|}
\hline $\overrightarrow{\dot{S}}$ & 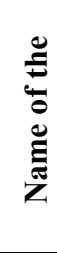 & 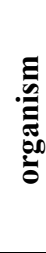 & $\begin{array}{l}\dot{0} \\
\dot{0} \\
\dot{z}\end{array}$ & 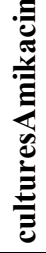 & لٍ & 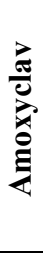 & 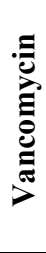 & 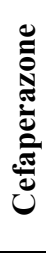 & 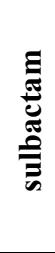 & نُ & : \\
\hline 1 & \multicolumn{2}{|c|}{ CONS } & 3 & 2 & 2 & 3 & 3 & 3 & & 1 & 2 \\
\hline 2 & \multicolumn{2}{|c|}{ COPS } & 1 & - & 1 & - & 1 & - & & 1 & - \\
\hline & \multicolumn{2}{|c|}{ Total } & 4 & 2 & 3 & 3 & 4 & & 2 & 2 & 2 \\
\hline
\end{tabular}

Gram positive isolates show maximum sensitivity to vancomycin, followed by Gentamicin, Amoxyclav, and cefeperazone-sulbactam.

Table No-4: Outcome.

\begin{tabular}{|c|c|c|c|}
\hline Outcome & Deaths & Survivals & Total \\
\hline No of cases & 10 & 64 & 74 \\
\hline Observation: & & & \\
\hline
\end{tabular}

Case fatality rate was $13.5 \%$

Table No.-5: Distribution of mortality according to culture positivity.

\begin{tabular}{|c|c|c|c|}
\hline & & Outcome & Total \\
\hline Culture & Deaths & Survivals & 12 \\
\hline Bacteriologically Positive & 4 & 8 & 62 \\
\hline Bacteriologically Negative & 6 & 56 & $\mathbf{7 4}$ \\
\hline Total & $\mathbf{1 0}$ & $\mathbf{6 4}$ & \\
\hline
\end{tabular}

Table No.-6: Distribution of mortality according to Gram-staining of organisms isolated in bacteriologically positive cases

\begin{tabular}{|c|c|c|c|}
\hline \multirow{2}{*}{ Gram-staining } & \multicolumn{3}{|c|}{ Out come } \\
\cline { 2 - 4 } & Deaths & Survivals & 8 \\
\hline Gram negative & 3 & 5 & 4 \\
\hline Gram positive & 1 & 3 & 12 \\
\hline Total & 4 & 8 & Total \\
\hline
\end{tabular}

3 deaths occurred in gram negative organisms. 1death occurred in gram positive organisms.

Table No.-7: Distribution of mortality according to organisms isolated in bacteriologically Positive cases

\begin{tabular}{|c|c|c|c|}
\hline Organism & & Outcome & Total \\
\hline & Deaths & Survivals & 4 \\
\hline E.coli & 2 & 2 & 3 \\
\hline Klebsiella & 1 & 2 & 3 \\
\hline CONS & 1 & 2 & 1 \\
\hline COPS & 0 & 1 & 1 \\
\hline Moraxella & 0 & 1 & $\mathbf{1 2}$ \\
\hline
\end{tabular}


E.coli was detected in 4 cases, out of that 2 cases died, and 2 cases survived. Klebsiella was detected in 3 cases, out of that 1 case died, 2 cases survived. CONS was detected in 3 cases, out of that 1 case died, 2 cases survived. COPS was detected in one case, which one survived. Moraxella was detected in one case, and that baby survived.

\section{Discussion}

This study was conducted at ASRAM, Eluru during study period of $1 \frac{1}{2}$ years i.e. from January 2014 to July 2015. Total 74 neonates below the age of 28 days with clinical suspicion of neonatal septicemia were included in this study.

Early onset septicemia i.e $<72$ hrs was present in 49 cases $(66 \%)$ and late onset septicemia was present in 25 cases (34\%). So early-onset septicemia was more common than late-onset septicemia, findings are consistent with other studies. In present study culture positivity rate was $18 \%$. P.jyothi et al[3] observed culture positivity rate of $19.2 \%$. Shrestha et al[5], Galhotra et al[6], Rajarshi Basu et al[4], Misra et al[7] observed culture positivity rate of $6.1 \%, 7.8 \%, 32.3 \%$, $39.6 \%, 65.2 \%$ respectively.Most common organism isolated in our study was E. coli followed by Klebsiella and CONS. Similarly E. coli were the commonest group of organisms isolated in other studies by N. Mehrotra [8] and Khatura et al.[9].

Chaturvedi et. al[10] and Nellain et. al[11] Found that Klebsiella, E.coli and Pseudomonas were commonly isolated organisms.

Meharban Singh[1] observed Klebsiella pneumonia (29.7\%), Staphylococcus aureus (14.7\%) E.coli (13.9\%) pseudomonas (9.2\%) were common pathogens in analysis from hospital based data collected by National Neonatal Perinatal Database Network from different countries in our country [2].

In present study gram-negative septicemia was more common than gram-positive septicemia. Similar to our studies observation were made by Galhotra et al[6], P. jyothi et. Al [3], Misra et.al[7].

Out of 8 culture positive cases of early-onset septicemia, Gram-negative organisms were detected in 6 cases. In present study Gram-negative organisms were common cause of early-onset septicemia. Shrestha et al[5] observed that E.coli were found to be most common pathogen in both early onset $(83.3 \%)$ and late onset septicemia
(52.3\%), staphylococcus was more common in late onset sepsis than early onset sepsis. Thus in present study case fatality rate was $13.5 \%$, consistent with other studies.

Rajarshi Basu et.al [4], Galhotra et.al [12] observed mortality rate of $20 \%, 7 \%$ respectively. Mathur et. al[12] Observed case fatality rate of $43 \%$. Moreno et. al [13] Observed case fatality rate of $31 \%$.

Present study shows that 8 cases $(16.3 \%)$ with early onset septicemia died, while 2 cases $(8 \%)$ with late onset septicemia died. In present study Mortality was higher in early onset septicemia. Mathur et. al[13] observed mortality of $64.5 \%$ when the onset of illness was early. High mortality in association with early-onset septicemia was reported by Rajarshi Basu et al[4] (72.73\%). In contrast to present study Galhotra et al observed higher mortality rate is observed in late onset neonatal sepsis $(59 \%)$ compared to early onset sepsis [12].

In present study mortality was higher in culture positive case compared to culture negative cases. Mortality among Gram negative and Gram positive organisms was $37.5 \% \& 25 \%$ respectively. So in present study mortality was higher in Gram negative septicemia. Khatua et. El [9]. Observed mortality in blood culture positive and negative group was $69 \%$ and $40.5 \%$ respectively. Higher mortality in culture positive group also recorded by other was due to invasion of blood stream by large number of bacteria. Khatua observed that mortality rate in Gram-negative septicemia was $78.5 \%$ and that of Gram-positive septicemia was $38.5 \%$. Berger et. al [14] Observed that the case fatality rate of episodes caused by Gram negative organisms was significantly higher than that of Gram-positive organisms. One major factor in the high mortality rates in Gram-negative septicemia is probably the emergence of drug resistant strains of these bacteria against the commonly used antibiotics. E.coli was detected in 4 cases out of that 2 died and 2 cases survived. Klebsiella was isolated in 3 cases out of that 1 case died and 2 survived. CONS was detected in 3 cases, 1 died 
and 2 survived. Khatua et. al [9] observed that mortality was $100 \%$ in babies having blood culture positive with Pseudomonas and Citrobacter. Mortality was found to be $88 \%$ in babies with culture positive with Klebsiella. Koutociby et. al [15] observed that babies with culture positive of Pseudomonas and Klebsiella had highest mortality of $71 \%$ and $50 \%$ respectively.

\section{Conclusions}

Clinical features of neonatal septicemia are nonspecific and vague and may be clinically indistinguishable from those occurring in noninfectious condition during neonatal period. Early-onset septicemia is more common than lateonset septicemia. Gram-negative septicemia is more common than Gram-positive septicemia. Gram-negative organisms are common cause of early-onset septicemia. Mortality is higher in earlyonset septicemia and Gram-negative septicemia.

Funding: Nil, Conflict of interest: None initiated, Perission from IRB: Yes

\section{References}

1. Singh Meharban. Care of Newborn, 8th edition. New Delhi. CBS Publications.Jan 2015; 281-294.

2. Report of National Neonatal Perinatal Database (National Neonatology Forum) 2002-2003.

3. P. Jyothi et al. Bacteriological profile of neonatal septicemia and antibiotic susceptibility pattern of the isolates. J Nat Sci Bio Med 2013 ;4(2):306-309.

4. Rajarshi Basu, Syamalkumar Bandyopadhyay et.al. Study on correlation between sepsis screening and blood culture in neonatal sepsis. IOSR journal of Dental and Medical Sciences (IOSR-JDMS) 2014; $13:(5)$ 52-56

5. Shrestha NJ et.al. Bacteriological profile of neonatal sepsis: A Hospital Based Study. J Nepal Paediatr Soc Jan-April2011; 31:(1) 1-5.
6. Shipra Galhotra et al. Clinico-bacteriological profile of neonatal septicemia in a tertiary care hospital. Journal of Mahatma Gandhi Institute of Medical sciences.2015;20:(2) 148-152.

7. Rabindra N Misra, Savita V Jadhav, Purbasha Ghosh, Nageswari Gandham, Kalpana Angadi, Chanda Vyawahare et. al. Role of sepsis screen in the diagnosis of neonatal sepsis. Medical journal of Dr. D.Y.Patil University.2013; 6:(3 ) 254-257.

8. Mehrotra $\mathrm{N}$ et al. Neonatal sepsis, correlation of maternal and neonatal factors to positive blood cultures. Indian pediatrics 1985;22;(4) 275-280.

9. Khatua SP, Das AK, Chatterjee BD, Khatua S, Ghose B, Saha A. Neonatal septicemia. Indian J Pediatr. 1986 Jul-Aug;53(4):509-14.

10. Chaturvedi P, Agrawal M, Narang P. Analysis of blood culture isolates from neonates of a rural hospital. Indian Paediatrics 1989 ; 26 : (5) 460-465

11. Nellian AR, Choudhury Panna, Shrinivasan S, Nalini P, Puri RK. A prospective study of bacterial infections in the newborn. Indian Journal of Pediatrics $1981 ; 48$ :(4) 427-431.

12. Mathur NB. Neonatal sepsis. Indian Pediatr. 1996 Aug;33(8):663-74.

13. Moreno MT, Vargas S, Poveda R, Sáez-Llorens $\mathrm{X}$. Neonatal sepsis and meningitis in a developing Latin American country. Pediatr Infect Dis J. 1994 Jun;13(6):516-20.

14. Berger A, Salzer HR, Weninger M, Sageder B, Aspöck C. Septicaemia in an Austrian neonatal intensive care unit: a 7-year analysis. Acta Paediatr. 1998 Oct;87(10):1066-9.

15. Koutouby A, Habibullah J. Neonatal sepsis in Dubai, United Arab Emirates. J Trop Pediatr. 1995 Jun;41(3):177-80.

\section{How to cite this article?}

Sahoo M.R, Arigela V, A Sirisha. Bacteriological spectrum and immediate outcome of neonatal sepsis in tertiary care centre in South India. J PediatrRes.2017;4(06):405-410.doi:10.17511/ijpr.2017.i06.09. 